\title{
SURFACE MICROMACHINED SILICON CARBIDE ACCELEROMETERS FOR GAS TURBINE APPLICATIONS
}

\author{
Russell G. DeAnna \\ U.S. Army Research Laboratory \\ Vehicle Technology Center \\ NASA Lewis Research Center \\ Cleveland, $\mathrm{OH} 44135$ \\ e-mail: Russell.G.Deanna@lerc.nasa.gov
}

\begin{abstract}
A finite-element analysis of possible silicon carbide $(\mathrm{SiC})$, foldedbeam, lateral-resonating accelerometers is presented. Results include stiffness coefficients, acceleration sensitivities, resonant frequency versus temperature, and proof-mass displacements due to centripetal acceleration of a blade-mounted sensor. The surface micromachined devices, which are similar to the Analog Devices@ Inc., (Norwood, MA) air-bag crash detector, are etched from $2-\mu \mathrm{m}$ thick, $3 \mathrm{C}-\mathrm{SiC}$ films grown at $1600 \mathrm{~K}$ using atmospheric pressure chemical vapor deposition (APCVD). The substrate is a $500 \mu \mathrm{m}$-thick, (100) silicon wafer. Polysilicon or silicon dioxide is used as a sacrificial layer. The finiteelement analysis includes temperature-dependent properties, shape change due to volume expansion, and thermal stress caused by differential thermal expansion of the materials. The finite-element results are compared to experimental results for a $\mathrm{SiC}$ device of similar, but not identical, geometry. Along with changes in mechanical design, blademounted sensors would require on-chip circuitry to cancel displacements due to centripetal acceleration and improve sensitivity and bandwidth. These findings may result in better accelerometer designs for this application.
\end{abstract}

\section{INTRODUCTION}

Condition monitoring is increasingly being used to provide information about the health of rotating machinery, as it can reveal the cause of potential problems and provide an early indication of incipient mechanical failures. Vibration is the most commonly measured parameter on rotating equipment, and vibration analysis typically detects faults due to imbalance, misalignment, bearing faults, and gearing problems. Most vibration measurements involve accelerometers or proximity probes located in the static frame on the casing or bearing housing. A vibration-monitoring system should consider the following items: selection of engine parts to be monitored and appropriate measuring locations; measuring sensors to be used; data evaluation procedures and criteria for deciding what is the significance of collected data; and vibration limits and reference values which establish the condition of the engine.

Permanently mounted sensors on the casing and bearing housings are commonly used to detect shaft-related vibration problems caused by bearing failures, misalignment, shaft cracks, structural resonance, unbalance, shaft bow, fluid-film and bearing instability, and rubs. Identification of the condition of the blading itself has not received much attention, although blading problems have been reported to rank among the most frequent engine faults (Mathioudakis, et al., 1989). Correlating engine outer-surface measurements to events in the engine interior is an indirect process because vibration measured at an axial location corresponding to a blade row is not necessarily dominated by the excitation of that row, but contain frequencies excited by the blade rows at other axial positions. A more direct measure of blade condition is desirable.

High-temperature strain gauges offer one of the few direct methods to measure blade vibratory stresses and frequencies. Strain gauges have been successful, but they are still limited by fatigue stresses and other issues. Compared to strain gauges, accelerometers don't have fatigue problems, are not as sensitive to temperature, and may be better at detecting blade failure since they measure motion instead of strain. For example, a strain gage may miss a blade vibration mode if the gage is not located in a region of strain. An accelerometer measures motion.

Required resolution for accelerometers on machinery running at 600-10,000 rpm may be only $80 \mu \mathrm{g}$ (Robinson et al., 1997). The upper range of acceleration may be $100 \mathrm{~g}$, and the blade may experience a 50,000-g centripetal acceleration. This requires a stiff suspension parallel to the centripetal acceleration and flexible suspensions in the directions of the acceleration to be measured. The fault frequencies to be caught are the first few multiples of the running speed, blade-pass frequencies, and gear-mesh frequencies for gearing systems. For most machinery, a bandwidth of $1.5 \mathrm{~Hz}$ to $1000 \mathrm{~Hz}$ catches all the normal vibrations. However, it is equally important to capture faults that initiate stress waves, such as fatigue cracks and impacting. Stress waves generally excite broad frequency bands, which are easiest to detect at frequencies exceeding the normal vibrations (i.e., at frequencies exceeding $1000 \mathrm{~Hz}$ ). Therefore, the bandwidth of an acceptable accelerometer is $1.5 \mathrm{~Hz}$ to 7 $\mathrm{kHz}$ for most rotating machinery.

Passive accelerometers rely on spring effects whereas active rebalance uses some form of closed-loop force generation to hold the proof mass in place. The required force-generation signal is proportional to the acceleration of the proof mass. Active rebalance schemes used in 
the past have included magnetic and electrostatic sensing and torquing. The spring constant of the rebalancing force is a prime determinant of an accelerometer's sensitivity. Feedback can also be used to cancel displacements due to centripetal acceleration and extend bandwidth and sensitivity, allowing optimization of sensitivity regardless of bandwidth. The useful bandwidth can be increased by a factor equal to the loop gain (Boser and Howe, 1996).

The most commonly used accelerometers in the industrial market are those using piezoelectric materials, but they are not widely accepted for high-volume installations because they are relatively expensive. Because piezoelectric accelerometers are sensitive only to dynamic changes in acceleration or stress, they are often called a.c.-responding sensors and as such cannot respond to static or d.c. acceleration. Undamped piezoelectric accelerometers typically have resonant frequencies between 10 and $30 \mathrm{kHz}$ and use the resistance change with strain of a semiconductor in cooperation with a proof mass. Piezoresistive devices are usually fabricated using bulk micromachining techniques and respond to static accelerations as well as dynamic excitations. In general, commercial undamped piezoresistive accelerometers are passive designs with off-chip circuitry, which is dictated by yield issues. A typical bulk-machined, cantilever-beam, piezoresistive device has a range of $100,000 \mathrm{~g}$, resonance frequency of $100 \mathrm{kHz}$, and a sensitivity of $0.75 \mu \mathrm{V} / \mathrm{g}$ (Ning, et al., 1995).

Surface micromachining of polysilicon has become established as a solution for a variety of sensing problems (Boser and Howe, 1996). Using only a few additional processing steps compatible with standard fabrication techniques and materials, mechanical sensing elements and the associated electronic interface circuits can be fabricated on a single die. Accelerometers made this way are being used in the automotive market as crash detectors and for dynamic vehicle control. Applications as vibration and shock detectors range from monitoring mechanical stress in airplane wings to recording mechanical shock of fragile shipping goods. The small feature size of surface micromachined structures renders it economical to place circuits and sensors on the same die. In contrast, bulk micromachining is fundamentally size-limited. Surface micromachining can implement a sensor, complete with all of the required signal conditioning, that is the same size as the bulk micromachined sensor alone. Surface micromachining has been used to make both piezoresistive and capacitive accelerometers.

There are a variety of folded-beam accelerometers fabricated using surface-micromachining techniques. Because of basic design considerations, folded-beam devices almost always have resonant frequencies between 500 and $5000 \mathrm{~Hz}$. This range, together with a very high $Q$, or amplification factor, necessitates damping both for selfprotection and to extend the useful frequency range. A nule of thumb suggests that the useful bandwidth is around one-third of the resonant frequency for undamped devices. Accelerometers are usually damped with air or silicone oil to a point near critical damping, and their useful frequency range will be about one-half of resonance. Sensitivity is inversely proportional to resonance frequency.

Surface micromachined capacitive accelerometers often have differential capacitance structures whose change in capacitance for a given displacement is first order for small deflections. These devices may have plates which function as actuators by using electrostatic forces allowing motion of the structure to be both sensed and induced. Symmetrical pairs of comb fingers provide differential capacitance plates only in the plane of the device. The out-of-plane direction would have only a single, parallel-plate capacitor. Electrostatic levitation can be used to compensate for the missing upper plate'.

The Analog-Devices( Inc. ADXL-50 is a silicon-based, surfacemicromachined, capacitive sensor with integrated circuitry. It uses four single beams and has anchor locations on the outside of a central proof mass. The entire chip is $3 \mathrm{~mm}$ by $3 \mathrm{~mm}$ in size, with the sensor region occupying only $7 \%$ of the total chip area. The proof mass is typically held $1.6 \mu \mathrm{m}$ above the substrate, with lateral features of similar size. The position of the mechanical structure above the substrate eriables sensors to move in three axes. The ability to sense the minute changes in capacitance that may result from moving silicon is simplified by the onchip circuitry, which reduces parasitic capacitance and interference from noise.

Micromachined accelerometers and the automation of the manufacturing process used to produce them have allowed the possibility of low-cost sensors. But the dynamic- and frequency-range of commercial devices are too low for general use in vibration monitoring. Micromachined accelerometers also consume more power than piezoelectric sensors. A typical specification for micromachined accelerometers is 5 VDC at 8-10 mA. Low-power piezoceramic (PZT) sensors are available with excitation requirements as low as 5 VDC at 0.3 $\mathrm{mA}$. At this time, piezoceramic devices are superior to the silicon micromachined accelerometers in terms of resolution, bandwidth, temperature range, ruggedness, and power consumption (Robinson et al., 1997). A typical double-cantilever, piezoresistive accelerometer has an output of $5 \mathrm{mV} / \mathrm{g} / \mathrm{volt}$ of bridge drive and resonance of $500 \mathrm{~Hz}$; a device with a resonance of $37 \mathrm{kHz}$ would have a sensitivity of $5 \mu \mathrm{V} / \mathrm{g} / \mathrm{volt}$ of bridge drive?

Deep reactive ion etching (DRIE) has also been used to form highaspect-ratio, force-balanced, capacitive accelerometers. The high aspect ratio achievable with this approach ( 25 ) allows for very high off-axis stiffness, large capacitance ( $3 \mathrm{pf}$ ) and a relatively heavy proof mass (43 $\mu \mathrm{g})$. Performance measurements show a bandwidth of $1 \mathrm{kHz}$, a sensitivity of $700 \mathrm{mV} / \mathrm{g}$, and a dynamic range of $44 \mathrm{~dB}$.

Silicon accelerometers are small enough for blade mounting, but sensors with internal electronics generally operate up to only $125^{\circ} \mathrm{C}$. This covers most industrial applications but falls far short of typical gasturbine applications. Silicon carbide $(\mathrm{SiC})$ has a higher elastic modulus ${ }^{3}$ and excellent high-temperature mechanical and electrical properties which makes it an attractive material for wide-temperature applications like the gas turbine. While the turbine section is likely too hot for $\mathrm{SiC}$ sensors and circuitry, most of the compressor stages are cool enough to permit a SiC sensor capable of $600^{\circ} \mathrm{C}$ operation. The accelerometer could be located in a cavity in the blade and sealed, and not be exposed to the gas path. The designs considered in this paper differ from the Analog Devices Inc. ADXL-50 mainly in the SiC material for the structural elements. Unfortunately, integration of $\mathrm{SiC}$ structures with $\mathrm{SiC}$ electronics will remain a future hope for some time since the mechanical elements are much further developed than the circuits.

\footnotetext{
'MEMS Accelerometer Elevates PC Game Control, Sensor Business Digest, vol. 8, no. 1, Nov., 1998.

2 Understanding Accelerometer Technology, IC Sensors, Inc., Milpitas, CA.

${ }^{3}$ Various groups have measured Young's modulus for SiC between 250 and 400 $\mathrm{GPa}$ compared to $160 \mathrm{GPa}$ for silicon.
} 


\section{FOLDED-BEAM ACCELEROMETER}

The advantages of lateral resonating devices with folded-beam suspensions include the capability of low spring constants in a small area, good flexibility in two directions, and high stiffness in the third. The folded beams also reduce the thermal stress due to differential thermal expansion between the substrate and suspended element. Singlepoint or closely spaced anchors reduce resonant frequency changes with temperature (DeAnna et al., 1999). Figure 1 shows a scanning-electronmicroscope (SEM) image of a SiC folded-beam resonator. Figure 2 shows a half-sketch with labels. The suspended elements comprising the shuttle, truss, and beams are attached to the substrate at four anchor locations. The comb-like, electrostatic fingers drive and sense the shuttle's lateral motion'. Gaps are typically $1 \mu \mathrm{m}$ with fingers 10 to 50 $\mu \mathrm{m}$ long. Since capacitance between the substrate and shuttle is inversely proportional to the gap for small displacements, maximum sensitivity requires minimum gap. The gap size is dictated by the sacrificial layer and must be chosen sufficiently large to prevent the moving element from touching the substrate due to residual-stress gradient and mechanical excitation.

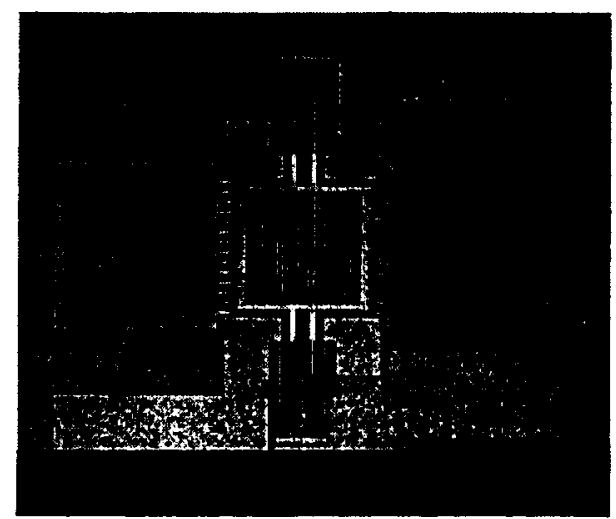

Figure 1. Scanning electron microscope image of a typical SiC lateral resonator.

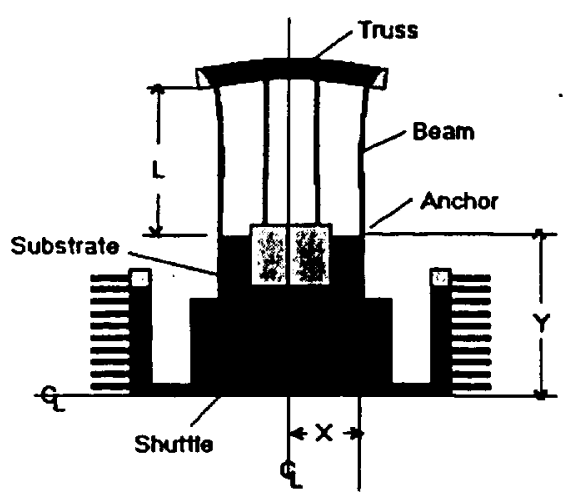

Figure 2. Geometry of the folded-beam structure.

\section{FINITE ELEMENT MODEL}

The ANSYS' finite-element package was used to predict stiffness coefficients, sensitivities, resonant-frequency versus temperature, and displacement under centripetal acceleration, for the devices shown in Fig. 3. One device (type I) has anchors close together and a small shuttle; the other (type II) has a large shuttle and widely spaced anchors. The designs were chosen to better understand the temperature-dependent behavior. The substrate between the anchors is included in the model because the anchors move according to the thermal expansion of the substrate. The sensitivity, $\Lambda$, in displacement per applied acceleration for the foldedbeam system is inversely proportional to the resonance frequency, $\omega_{n}$, according to

$$
\Lambda=\frac{1}{\omega_{n}^{2}} \propto \frac{M}{k}
$$

where $M$ is the effective mass and $k$ the stiffness. The resonant frequency is (Biebl et al., 1995)

$$
\omega_{n}^{2}=\frac{24 E I}{L^{3} M}
$$

where $E$ is Young's modulus, $I$ is the cross-sectional moment of inertia of the beams, and $\mathrm{L}$ is the beam length. As mentioned previously, the useful bandwidth is some fraction of $\omega_{n}$. The SiC layer is $2 \mu \mathrm{m}$ thick. Beams are $100 \mu \mathrm{m}$ long by $2 \mu \mathrm{m}$ wide in the type-I device and $150 \mu \mathrm{m}$ by $3 \mu \mathrm{m}$ in the type-ll device. Including the comb fingers on the substrate, the devices occupy a chip area less than 500 by $500 \mu \mathrm{m}$.
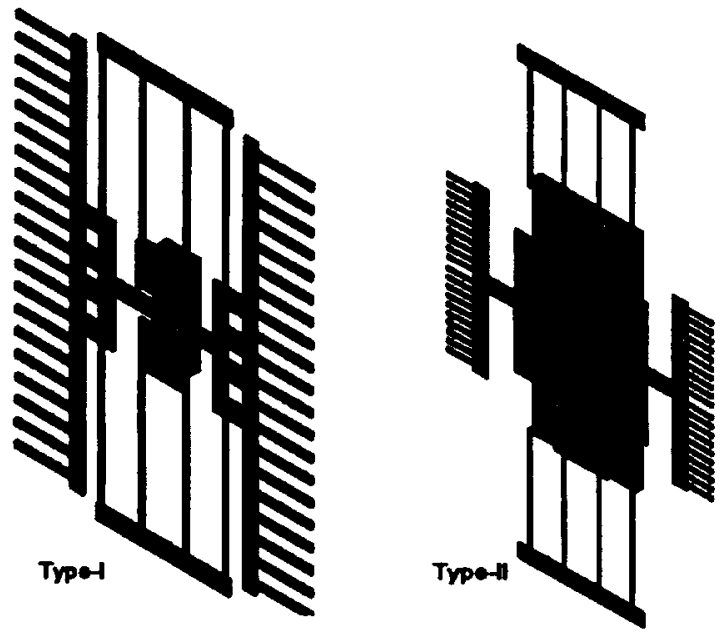

Figure 3. Finite-element model geometries of the type-I and type-II devices.

\footnotetext{
'The comb fingers could also be parallel to the beams with lateral motion changing the gap between fingers.
}

'ANSYS, Inc., Canonsburg, PA 15317. 
Table 1. Room-temperature stifiness coefficients and acceleration sensitivity at $300 \mathrm{~K}$.

\begin{tabular}{|c|c|c|c|}
\hline \multirow{2}{*}{ Device } & \multicolumn{3}{|c|}{ Stiffness $(\mathrm{N} / \mathrm{m})$} \\
\cline { 2 - 4 } & Lateral Direction $(\mathrm{x})$ & Vertical Direction $(\mathrm{y})$ & Out of Plane $(\mathrm{z})$ \\
\hline Type I & 6.92 & 1720 & 5.65 \\
\hline Type II & 6.62 & 7100 & 2.39 \\
\hline & \multicolumn{3}{|c|}{ Sensitivity $(\mu \mathrm{m} / \mathrm{g})$} \\
\hline Type I & $1.9 \times 10^{-5}$ & $7.7 \times 10^{-4}$ & $2.3 \times 10^{-5}$ \\
\hline Type II & $7.7 \times 10^{-5}$ & $7.2 \times 10^{-4}$ & $2.1 \times 10^{-4}$ \\
\hline
\end{tabular}

\section{RESULTS}

The stiffiness coefficients shown in Table 1 indicate the magnitude of the electrostatic drive force necessary to deflect the floating element or, combined with the moving mass, the sensitivity to acceleration. The stiffiness coefficients were obtained by computing the shuttle displacement for a $1 \mu \mathrm{N}$ force applied at the center of the shuttle. As expected, the devices are stiff in the vertical direction parallel to the beams and flexible in the directions perpendicular to the beams. The type-Il device has a higher sensitivity because of the more massive shuttle. The moving masses are $0.132 \mu \mathrm{g}$ and $0.510 \mu \mathrm{g}$ for the type-I and type-II devices, respectively. Shuttle displacements would be on the order of $100 \AA$ for a $100-g$ acceleration. Sensitive circuitry would be necessary to detect the associated capacitance changes. A thicker $\mathrm{SiC}$ layer would add mass and increase the sensitivities in all directions and the stiffness in the $\mathrm{z}$ direction. Of course, this would lower the resonant frequency.

Table 2 lists room-temperature material properties used in the analysis. Temperature-dependent thermal expansion coefficients for $\mathrm{Si}$ and $\mathrm{SiC}$ were used (Reeber and Wang 1996a, Reeber and Wang 1996b). These values are highly non-linear, and only the room-temperature values are given in the table. Since the stiffness coefficient is proportional to Young's modulus, sensitivities for other materials can be scaled from Eq. (1), and resonant frequencies from Eq. (2). The temperature coefficient of Young's modulus is constant and can be used to compute the decrease in $\mathrm{E}$ at any temperature (Biebl et al., 1995).

The modal results were obtained for temperatures from $300 \mathrm{~K}$ to $1300 \mathrm{~K}$ and include temperature-dependent properties, thermal stress, and thermal expansion. They neglect squeeze-film damping due to gas pressure. The first four mode shapes for the two types of devices are shown in Figs. 4 and 5 . In both cases, the lateral mode is the third, the second mode is out of the plane, and modes one and four are rocking or tilting. Depending on orientation, the blade motion may excite some of these modes.
The room-temperature resonant frequencies for the devices are given in Table 3. The sensors' frequency bandwidths are sufficient to
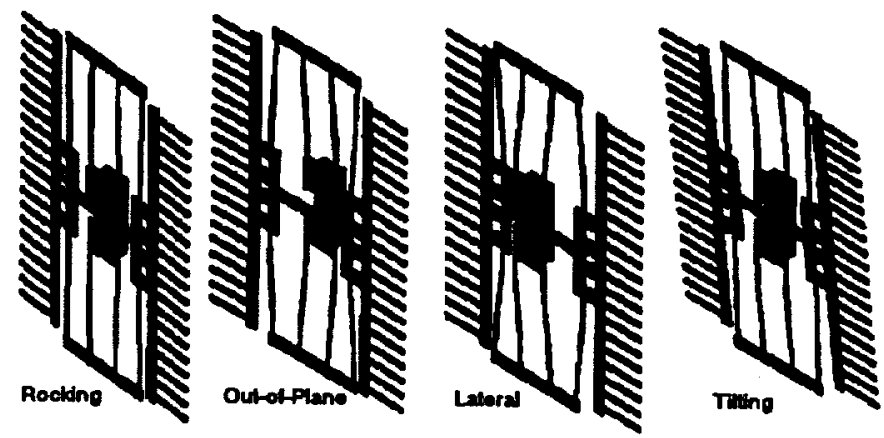

Figure 4. Mode shapes one through four for type-I device.
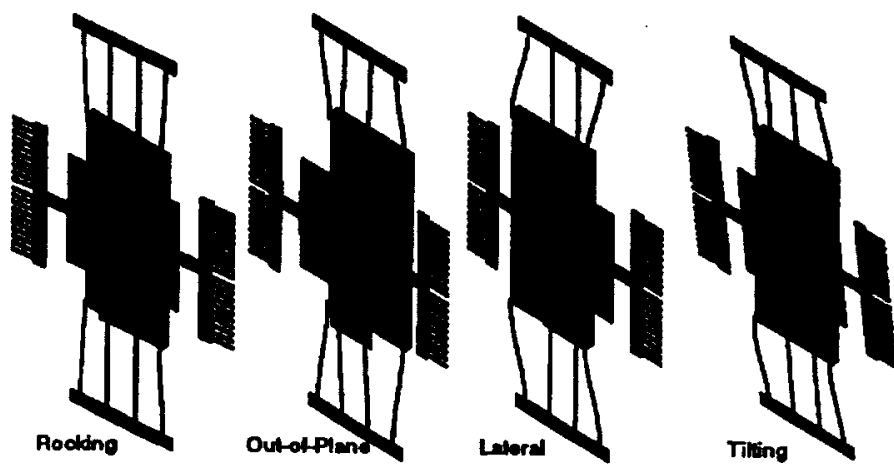

Figure 5. Mode shapes one through four for type-II device.

Table 2. Room-temperature material properties used in the analysis.

\begin{tabular}{|c|c|c|c|c|}
\hline Material & Density $\left(\mathrm{kg} / \mathrm{m}^{3}\right)$ & $\begin{array}{c}\text { Young's modulus } \\
\text { coefficient }\left(10^{-6} / \mathrm{K}\right)\end{array}$ & $\begin{array}{c}\text { Thermal expansion } \\
\text { coefficient }\left(10^{-6} / \mathrm{K}\right)\end{array}$ & $\begin{array}{c}\text { Young's modulus } \\
(\mathrm{GPa})\end{array}$ \\
\hline $\mathrm{SiC}$ & 3230 & -46 & 2.25 & 250 \\
\hline $\mathrm{Si}$ & 2320 & -80 & 2.6 & 160 \\
\hline $\mathrm{SiO}_{2}$ & 2000 & -46 & 0.734 & 100 \\
\hline
\end{tabular}

Table 3. Room-temperature resonant frequencies for modes one through four.

\begin{tabular}{|c|c|c|c|c|}
\hline \multirow{2}{*}{ Device } & \multicolumn{4}{|c|}{ Resonant Frequency (kHz) } \\
\cline { 2 - 5 } & Mode I & Mode II & Mode III & Mode IV \\
\hline Type-I & 23 & 39 & 43 & 49 \\
\hline Type-II & 7 & 12 & 21 & 25 \\
\hline
\end{tabular}


measure blade accelerations below $5000 \mathrm{~Hz}$. Bandwidth could be traded for sensitivity by increasing the shuttle mass; however, substantially lowering the resonant frequency compromises the reliability of the device, in particular its robustness to mechanical shock, and increases the probability of the proof mass sticking to the substrate. Dropping a sensor from a bench top can result in a 200 to $400 \mathrm{~g}$ shock. Hence, shock protection is usually necessary. There is also the problem of damping. Evacuating the chamber around the proof mass allows the sensor to resonate in the out-of-plane direction but it eliminates the squeeze-film damping in gaps between the proof mass and the supporting structure. Vacuum packaging also eliminates the noise caused by Brownian motion and has been shown to reduce thermal noise by several orders of magnitude (Boser and Howe 1996). Overdamping decreases the useful bandwidth but offers protection from excessive displacements at resonance. For underdamped devices, the accelerometer is more prone to signal distortion and breakage when excited near the peak response.

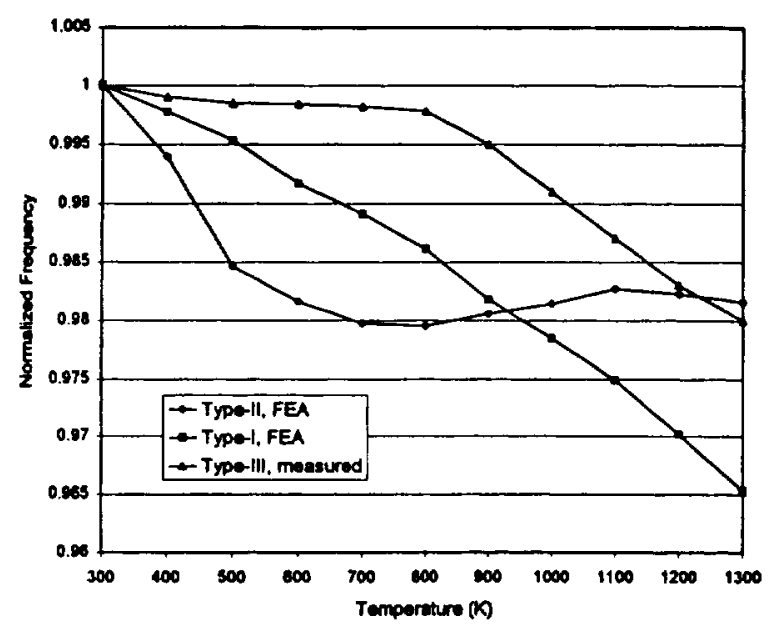

Figure 6. Resonant frequency of the lateral modes normalized with the room-temperature frequency plotted versus temperature.

Figure 6 shows the resonant frequency of the lateral mode normalized by the room-temperature frequency as a function of temperature. Also plotted is the experimental data for the type-III device as shown in Fig. I (Fleischman et al., 1998). Like the type-Il device, the type-III device has a large shuttle and widely-spaced anchors.

Most of the change in resonant frequency of the type-I device can be attributed to the decrease in the temperature coefficient of Young's modulus, which is $4.6 \%$ for $\mathrm{SiC}$ over the $1000 \mathrm{~K}$ temperature range. The resonant frequency drop is less because volume expansion and thermal stress increases resonant frequency. The interesting behavior of the typeII device is caused by the thermal stress in the beams due to the widelyspaced anchor locations. The frequency drops by $2 \%$ between 300 and $700 \mathrm{~K}$, then rises slightly up to $1300 \mathrm{~K}$. The shape is dictated by the thermal expansion coefficients of $\mathrm{Si}$ and $\mathrm{SiC}$. Below $700 \mathrm{~K}$ the value for $\mathrm{Si}$ is greater than that for $\mathrm{SiC}$. The opposite is true above $700 \mathrm{~K}$. The overall decrease in frequency for the type-II device matches the experimental result for the type-lll device, though the shape of the curves are different. In all cases, the decrease in resonant frequency is accompanied by a corresponding increase in sensitivity according to Eq.(1). The sensitivity increase may or may not be critical. The resonant frequency shift would be important if the sensor were designed to operate at resonance. The results suggest that the type-I device with closelyspaced anchor locations has a more predictable behavior with temperature and would be easier to compensate for with circuitry.

The maximum displacements with a centripetal acceleration of 18 $\mathrm{kg}$ are given in Table 4. The results represent the maximum displacement found anywhere on the device and cannot be obtained from the sensitivities in Table 1 which are for the center of the shuttle. For example, the maximum out-of-plane displacements for a rotation $\omega_{x}$ occurs at the ends of the comb fingers. The displacements for other accelerations can be interpolated or extrapolated from these data. The 18 $\mathrm{kg}$ acceleration corresponds to the sensor on a blade offset by $0.5 \mathrm{~m}$ in the direction along the $y$-axis from the centerline of a shaft rotating at $6000 \mathrm{rpm} . \omega_{z}$ creates a centripetal acceleration parallel to the beams; $\omega_{x}$ creates an acceleration normal to the plane of the device.

The extra mass of the type-II device improves sensitivity but also causes large displacements with certain orientations. When the direction of centripetal acceleration is parallel to the beams, the displacements are less than $1 \mu \mathrm{m}$. Figure 7 shows the 2 displacement of

Table 4. Sensor maximum displacements due to centripetal acceleration.

\begin{tabular}{|c|c|c|c|c|}
\hline \multirow{2}{*}{ Device } & $\begin{array}{c}\text { Angular Velocity } \\
(\mathrm{rad} / \mathrm{s})\end{array}$ & \multicolumn{3}{|c|}{ Maximum Displacement $(\mu \mathrm{m})$} \\
\cline { 3 - 5 } & $\omega_{x}=600$ & \pm Lateral $(\mathrm{x})$ & Vertical $(\mathrm{y})$ & Out-of-plane $(\mathrm{z})$ \\
\hline \multirow{3}{*}{ Type-I } & $\omega_{z}=600$ & \pm 0.8 & \pm 0.8 & +6.9 \\
\cline { 2 - 5 } & $\omega_{x}=600$ & \pm 1.0 & \pm 0.3 & +0.01 \\
\hline \multirow{2}{*}{ Type-II } & $\omega_{z}=600$ & $+0.3,-0.5$ & $+0.5,-0.7$ & +71.6 \\
\cline { 2 - 5 } & & \pm 0.9 & $+0.3,-0.7$ & -0.14 \\
\hline
\end{tabular}




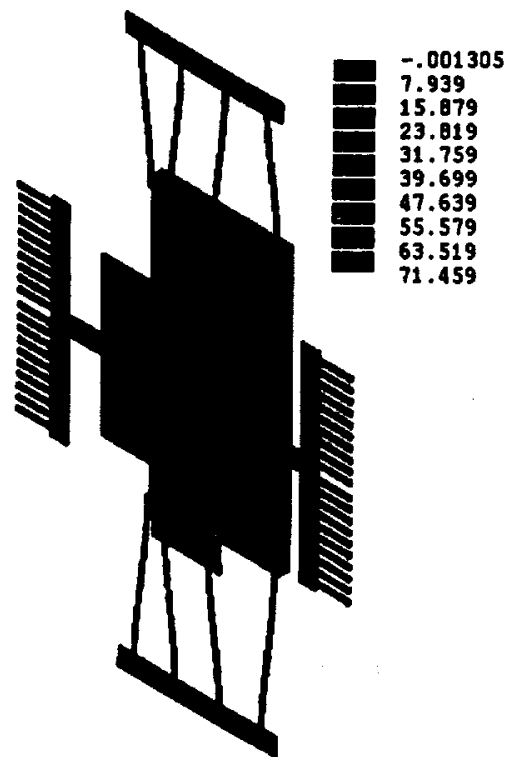

Figure 7. Out-of-plane displacement of type-II device due to angular velocity $\omega_{x}$.

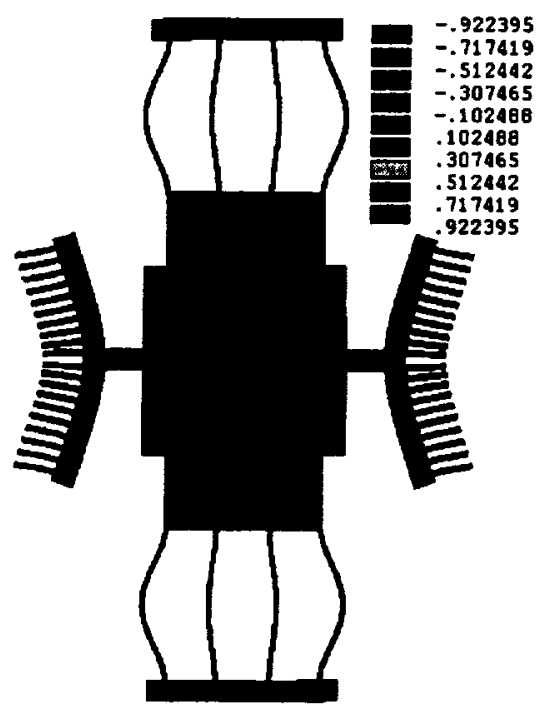

Figure 8. Horizontal displacement of type-II device due to angular velocity $\omega_{2}$.

the type-II device under the angular velocity $\omega_{x}$. Figure 8 shows the $x$ displacement of the device with an angular velocity $\omega_{z}$. The comb fingers and beams bend about $1 \mu \mathrm{m}$. Circuitry may be required to cancel these displacements. The $x$ - and $z$-axes' displacements could be canceled with electrostatic forces. The $y$-axis' displacements could not be canceled using a conventional lateral resonator. A design with comb fingers on all four sides of the shuttle would be necessary.

\section{CONCLUSIONS}

Two particular folded-beam resonators were considered for measuring compressor-blade accelerations. The analysis considered SiC sensors for wide-temperature applications, but the results can be applied to $\mathrm{Si}$ devices with the proper scaling and material properties. The bandwidth of the devices exceeds $5000 \mathrm{~Hz}$. Shuttle mass could be increased to trade bandwidth for sensitivity. Blade-mounted sensors were considered since this is the most challenging application. The high centripetal accelerations associated with rotating blades impose severe constraints on the sensor's mechanical elements. The displacements due to the centripetal accelerations would likely need to be cancelled using on-chip circuitry. A design with comb fingers on all sides of the shuttle is recommended to enable cancellation of acceleration in all three directions. Susceptibility to packaging stress will be complicated by the large $\mathbf{g}$ forces. This will also impose restrictions on packaging, wire bonding and mounting. In wide-band temperature applications, an onchip temperature sensor may be necessary to account for sensitivity changes. These results may be used to design improved accelerometers for gas-turbine applications.

\section{ACKNOWLEDGEMENTS}

I would like to thank C. A. Zorman, S. Roy, and A. J. Fleischman of Case Western Reserve University for valuable discussions on the behavior of the lateral resonating devices.

\section{REFERENCES}

Biebl, M., Brandl, G., and Howe, R. T., 1995, "Young's Modulus of in Situ Phosphons-Doped Polysilicon," The $8^{\text {th }}$ International Conference on Solid-State Sensors and Actuators, and Eurosensors IX, Stockholm, Sweden.

Boser, B. E. and Howe, R. T., 1996, "Surface Micromachined

Accelerometers," IEEE J. of Solid-State Circuits, vol. 31, no. 3.

DeAnna, R. G., Roy, S., Zorman, C. A., and Mehregany, M., 1999, "SiC Lateral Resonant Devices for High Temperature Applications," to be presented at Modeling and Simulation of Microsystems.

Fleischman, A. J., Roy, S., Zorman, C. A., and Mehregany, M., 1998, "Behavior of Polycrystalline SiC and Si Surface-Micromachined Lateral Resonant Structures at Elevated Temperatures," Proc. of the IEEE Intl. Conf. On Silicon Carbide, III-Nitrides, and Related Matls., ICSCIII-N 97, pp. 889-892.

Mathioudakis, K., Loukis, E., and Papailiou, K. D., 1989, “Casing Vibration and Gas Turbine Operating Conditions," ASME-89-GT-78, Gas Turbine and Aeroengine Congress and Exposition.

Ning, Y., Loke, Y., and McKinnon, G., 1995, "Fabrication and characterization of high g-force, silicon piezoresistive accelerometers," Sensors and Actuators A, vol. 48, pp. 55-61.

Reeber, R. R. and Wang, K., 1996a, "Thermal expansion and lattice parameters of group IV semiconductors," Mat. Chem. and Phy., vol. 46, pp. 259-264.

Reeber, R. R. and Wang, K., 1996b, "Thermal Expansion of $\beta-S i C$, GaP and InP," Mater. Res. Soc. Symp. Proc., vol. 410, pp. $211-216$.

Robinson, J. C., Rybak, J. M., and Saller, E. R., 1997, “Using Accelerometers to Monitor Complex Machinery Vibration," Sensors, June. 